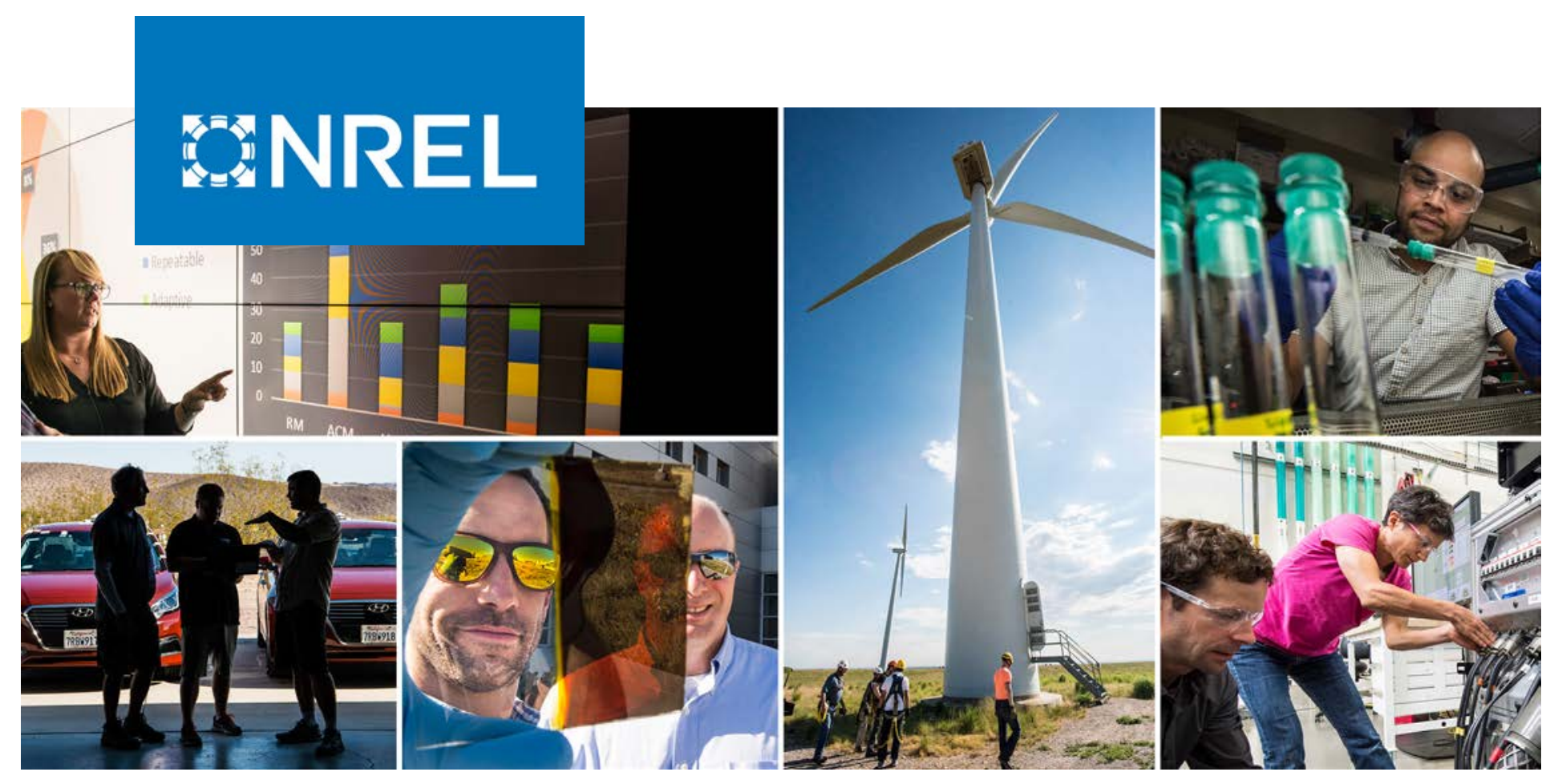

\title{
Graph Laplacian Spectrum and Primary Frequency Regulation
}

\section{Preprint}

Linqi Guo, ${ }^{1}$ Changhong Zhao, ${ }^{2}$ and Steven H. Low ${ }^{1}$

1 California Institute of Technology

2 National Renewable Energy Laboratory

Presented at the IEEE Conference on Decision and Control Miami, Florida

December 17-19, 2018

NREL is a national laboratory of the U.S. Department of Energy

Office of Energy Efficiency \& Renewable Energy

Operated by the Alliance for Sustainable Energy, LLC

This report is available at no cost from the National Renewable Energy Laboratory (NREL) at www.nrel.gov/publications.

\section{Conference Paper}

NREL/CP-5D00-71124

February 2019 


\title{
GNREL
}

\section{Graph Laplacian Spectrum and Primary Frequency Regulation}

\section{Preprint}

\author{
Linqi Guo, ${ }^{1}$ Changhong Zhao, ${ }^{2}$ and Steven H. Low ${ }^{1}$
}

1 California Institute of Technology

2 National Renewable Energy Laboratory

\section{Suggested Citation}

Guo, Linqi, Changhong Zhao, and Steven H. Low. 2019. Graph Laplacian Spectrum and Primary Frequency Regulation: Preprint. Golden, CO: National Renewable Energy Laboratory. NREL/CP-5D00-71124. https://www.nrel.gov/docs/fy19osti/71124.pdf.

(C) 2019 IEEE. Personal use of this material is permitted. Permission from IEEE must be obtained for all other uses, in any current or future media, including reprinting/republishing this material for advertising or promotional purposes, creating new collective works, for resale or redistribution to servers or lists, or reuse of any copyrighted component of this work in other works.

NREL is a national laboratory of the U.S. Department of Energy Office of Energy Efficiency \& Renewable Energy Operated by the Alliance for Sustainable Energy, LLC

This report is available at no cost from the National Renewable Energy Laboratory (NREL) at www.nrel.gov/publications.

Contract No. DE-AC36-08GO28308
Conference Paper

NREL/CP-5D00-71124

February 2019

National Renewable Energy Laboratory 15013 Denver West Parkway Golden, CO 80401

303-275-3000 • www.nrel.gov 


\section{NOTICE}

This work was authored in part by the National Renewable Energy Laboratory, operated by Alliance for Sustainable Energy, LLC, for the U.S. Department of Energy (DOE) under Contract No. DE-AC36-08GO28308. Funding provided by U.S. Department of Energy Office of Energy Efficiency and Renewable Energy Solar Energy Technologies Office. The views expressed herein do not necessarily represent the views of the DOE or the U.S. Government. The U.S. Government retains and the publisher, by accepting the article for publication, acknowledges that the U.S. Government retains a nonexclusive, paid-up, irrevocable, worldwide license to publish or reproduce the published form of this work, or allow others to do so, for U.S. Government purposes.

This report is available at no cost from the National Renewable Energy Laboratory (NREL) at www.nrel.gov/publications.

U.S. Department of Energy (DOE) reports produced after 1991 and a growing number of pre-1991 documents are available free via www.OSTI.gov.

Cover Photos by Dennis Schroeder: (clockwise, left to right) NREL 51934, NREL 45897, NREL 42160, NREL 45891, NREL 48097, NREL 46526.

NREL prints on paper that contains recycled content. 


\title{
Graph Laplacian Spectrum and Primary Frequency Regulation
}

\author{
Linqi Guo, Changhong Zhao, and Steven H. Low
}

\begin{abstract}
We present a framework based on spectral graph theory that captures the interplay among network topology, system inertia, and generator and load damping in determining the overall grid behavior and performance. Specifically, we show that the impact of network topology on a power system can be quantified through the network Laplacian eigenvalues, and such eigenvalues determine the grid robustness against lowfrequency disturbances. Moreover, we can explicitly decompose the frequency signal along scaled Laplacian eigenvectors when damping-intertia ratios are uniform across buses. The insight revealed by this framework suggests the reason why load-side participation in frequency regulation not only makes the system respond faster but also helps lower the system nadir after a disturbance. Finally, by presenting a new controller specifically tailored to suppress high-frequency disturbances, we demonstrate that our results can provide useful guidelines in the controller design for load-side primary frequency regulation. We simulate the improved controller on the IEEE 39-bus New England interconnection system to illustrate its robustness against highfrequency oscillations compared to the conventional droop control and a recent controller design.
\end{abstract}

\section{INTRODUCTION}

The electric grid is one of the largest and most complex engineering achievements, with numerous interconnected components that interact in an intricate fashion. Without detailed simulation and computation, it is hard to infer how the change to part of the system affects the overall grid behavior and performance [1], [2]. For example, one can argue that the connectivity in the grid helps average the power demand imbalance in the network, and therefore adding more connectivity should reduce overall system oscillation. On the other hand, one can also argue that more connectivity means faster propagation of disturbances throughout the network, and

Linqi Guo and Steven H. Low are with the Department of Computing and Mathematical Sciences, California Institute of Technology, Pasadena, CA, 91125. Email: \{lguo, slow $\}$ daltech.edu. Changhong Zhao is with the National Renewable Energy Laboratory, Golden, CO, 80401. Email: Changhong. Zhao@nrel.gov.

The authors thank Janusz Bialek and Oleg Khamisov from Skoltech for helpful discussions. This work was supported by Resnick Research Fellowship, Linde Institute Research Award, NSF grants through CCF 1637598, ECCS 1619352, CNS 1545096, ARPA-E grant through award DE-AR0000699 (NODES) and GRID DATA, DTRA through grant HDTRA 1-15-1-0003 and Skoltech through collaboration agreement 1075-MRA. The work of C. Zhao was also supported by the U.S. Department of Energy under Contract No. DEAC36-08GO28308 with Alliance for Sustainable Energy, LLC, the Manager and Operator of the National Renewable Energy Laboratory, as well as Award No. DE-EE0007998. Funding provided by Advanced Research Projects Agency-Energy (ARPA-E) and U.S. Department of Energy Office of Energy Efficiency and Renewable Energy Solar Energy Technologies Office.

The U.S. Government retains and the publisher, by accepting the article for publication, acknowledges that the U.S. Government retains a nonexclusive, paid-up, irrevocable, worldwide license to publish or reproduce the published form of this work, or allow others to do so, for U.S. Government purposes. therefore adding more connectivity should decrease system stability. Both arguments seem plausible, but they lead to (apparently) opposite conclusions. A corollary of our result in Section III will clarify this paradox.

The increasing penetration levels of renewable generation make such inferences even more difficult because it adds complexity to the system dynamics and increases volatility on the grid. For instance, one popular approach to maintaining system stability in this new era is to integrate load-side participation [3]-[8], which helps stabilize the system in a more responsive and scalable fashion and improves the system transient behavior [9]-[11]; however, so far such benefits are mostly observed through simulation or experimental evaluation [6], [8]-[14] and it is less clear how and why the load-side controllers affect (and improve) the system performance. There has also been work on improving the dynamic performance of the power system through controlling power electronics or loads. An example is iDroop [15], which is designed by analyzing the $\mathcal{H}_{2}$ norm. Such controllers usually make power system dynamics more sophisticated and uncertain and hence make it hard to obtain a stability guarantee [16]. Even more challenging but also more useful in applications is understanding how we should optimally choose the control parameters subject to certain performance goals in the controller design. See [17] for extensive references to recent literature on frequency control.

In this work, we present a framework that captures the interplay among network topology, system inertia, generator damping, and transmission line susceptance. Because of its combinatorial nature, the impact of network topology on a power system is usually hard to reason. Through this framework, we show that such impacts can be quantified using the network Laplacian eigenvalues. Our contributions can be summarized as follows: a) We show that whether the system oscillates or not in a particular supply-and-demand scenario is determined by how strong the damping is compared to the network connectivity in the "corresponding" direction. b) Adding network connectivity increases the system robustness against disturbances of relatively low frequency, but its benefit in suppressing high-frequency oscillation is limited. c) The network Laplacian eigenvalues reveal the set of harmonics that are amplified by the network. d) This framework unifies the optimization over many system and control parameters and captures the trade-offs among different design goals, which allows us to systematically improve and refine controller designs. Within this framework, we can devise a quantitative explanation on why load-side participation helps improve system behavior in both the transient and steady states. 
The rest of this paper is organized as follows. In Section II, we review the system model and relevant concepts from spectral graph theory. In Section III, we present our characterization of the system response in both the time and Laplace domain. The practical interpretations of our results are given in Section IV. In Section V, we quantify the benefits of loadside controllers and present a new controller systematically designed and specifically tailored to suppress high-frequency oscillation. In Section VI, we simulate the improved controller on the IEEE 39-bus New England interconnection test bed and illustrate its robustness against measurement noise and highfrequency oscillation in injection. We conclude in Section VII.

\section{NETWORK MODEL}

In this section, we present the system model as adopted in [10] and review relevant concepts from spectral graph theory.

Let $\mathbb{R}$ and $\mathbb{C}$ denote the set of real and complex numbers, respectively. We reserve uppercase symbols such as $A, B, C$ for matrices. For matrices $A, B$ with proper dimensions, $[A B]$ means the concatenation of $A, B$ in a row, and $[A ; B]$ means the concatenation of $A, B$ in a column. A variable without subscript usually denotes a vector with appropriate components, e.g., $\omega=\left(\omega_{j}, j \in \mathcal{N}\right) \in \mathbb{R}^{|\mathcal{N}|}$. For a timedependent signal, $\omega(t)$, we use $\dot{\omega}$ to denote its time derivative $\frac{d \omega}{d t}$. The identity matrix of dimension $n \times n$ is denoted as $I_{n}$. The column vector of length $n$ with all entries being 1 is denoted as $\mathbf{1}_{n}$. The imaginary unit $\sqrt{-1}$ is denoted as $\mathrm{j}$.

We use the graph $\mathcal{G}=(\mathcal{N}, \mathcal{E})$ to describe the power transmission network, where $\mathcal{N}=\{1, \ldots, n\}$ is the set of buses, and $\mathcal{E} \subset \mathcal{N} \times \mathcal{N}$ denotes the set of transmission lines. The terms bus/node and line/edge are used interchangeably in this paper. We assume without loss of generality that $\mathcal{G}$ is connected and simple. An edge in $\mathcal{E}$ is denoted either as $e$ or $(i, j)$. We further assign an arbitrary orientation over $\mathcal{E}$ so that if $(i, j) \in \mathcal{E}$, then $(j, i) \notin \mathcal{E}$.

Let $n, m$ be the number of buses and transmission lines, respectively. The incidence matrix of $\mathcal{G}$ is the $n \times m$ matrix $C$, defined as:

$$
C_{j e}= \begin{cases}1 & \text { if node } j \text { is the source of } e \\ -1 & \text { if node } j \text { is the target of } e \\ 0 & \text { otherwise }\end{cases}
$$

For each bus $j \in \mathcal{N}$, we denote its frequency deviation as $\omega_{j}$, and we denote the inertia constant as $M_{j}>0$. The symbol $P_{j}^{m}$ is overloaded to denote the mechanical power injection if $j$ is a generator bus, and denote the aggregate power injection from uncontrollable loads if $j$ is a load bus. For a generator bus, we model the droop control as $D_{j} \omega_{j}$ with $D_{j} \geq 0$, and for load buses, we use the same symbol to denote the aggregated frequency sensitive load. For each transmission line $(i, j) \in \mathcal{E}$, we denote as $P_{i j}$ the branch flow deviation, and we denote as $B_{i j}$ the line susceptance, assuming voltage magnitudes are 1 per unit (pu). With such notations, the swing and network dynamics are given by:

$$
\begin{array}{rlrl}
M_{j} \dot{\omega}_{j} & =-D_{j} \omega_{j}-d_{j}+P_{j}^{m}-\sum_{e \in \mathcal{E}} C_{j e} P_{e}, \quad j \in \mathcal{N} \\
\dot{P}_{i j} & =B_{i j}\left(\omega_{i}-\omega_{j}\right), & (i, j) \in \mathcal{E}
\end{array}
$$

We refer the readers to [10] for more detailed justification and derivation of this model.

Using $x$ to denote the system state $x=[\omega ; P]$, and putting $M, D$, and $B$ as the diagonal matrices with $M_{j}, D_{j}$, and $B_{i j}$ as the diagonal entries, respectively, we can rewrite the system dynamics (1) in the state-space form:

$$
\dot{x}=\left[\begin{array}{cc}
-M^{-1} D & -M^{-1} C \\
B C^{T} & 0
\end{array}\right] x+\left[\begin{array}{c}
M^{-1} \\
0
\end{array}\right]\left(P^{m}-d\right)
$$

We refer to the matrix:

$$
A=\left[\begin{array}{cc}
-M^{-1} D & -M^{-1} C \\
B C^{T} & 0
\end{array}\right]
$$

as the system matrix. We emphasize that the variables $[\omega ; P]$ denote deviations from their nominal values so that $x(t)=0$ means the system is in its nominal state at time $t$.

For any node $i \in \mathcal{N}$, we denote the set of its neighbors as $N(i)$. The (susceptance-weighted) graph Laplacian matrix of $\mathcal{G}$ is the $n \times n$ symmetric matrix $L=C B C^{T}$, which is explicitly given by:

$$
L_{i j}= \begin{cases}-B_{i j} & i \neq j,(i, j) \in \mathcal{E} \text { or }(j, i) \in \mathcal{E} \\ \sum_{k \in N(i)} B_{i k} & i=j \\ 0 & \text { otherwise }\end{cases}
$$

It is well known that if the graph $\mathcal{G}$ is connected, then $L$ has rank $n-1$, and any principal minor of $L$ is invertible [18]. For any vector $x \in \mathbb{R}^{n}$, we have:

$$
x^{T} L x=\sum_{(i, j) \in \mathcal{E}} B_{i j}\left(x_{i}-x_{j}\right)^{2} \geq 0
$$

This implies that $L$ is a positive semidefinite matrix and thus diagonalizable. We denote its eigenvalues and corresponding orthonormal eigenvectors as $0=\lambda_{1}<\lambda_{2} \leq \cdots \leq \lambda_{n}$ and $v_{1}, v_{2}, \cdots, v_{n}$. Note that we always have $v_{1}=\frac{1}{\sqrt{n}} \mathbf{1}_{n}$, a uniform vector. When the matrix $L$ has repeated eigenvalues, for each repeated eigenvalue $\lambda_{i}$ with multiplicity $m_{i}$, the corresponding eigenspace of $L$ always has dimension $m_{i}$, hence an orthonormal basis consisting of eigenvectors of $L$ exists (yet such bases are not unique). We assume that one of the possible orthonormal bases is chosen and fixed throughout the paper. For any vector $s \in \mathbb{R}^{n}$, we write its spectral decomposition as $s=\sum_{i} \hat{s}_{i} v_{i}$, with $\hat{s}_{i} \in \mathbb{R}$.

In the sequel, we further assume that the buses are homogeneous in the sense that $M=\mu I_{n}$ and $D=\delta I_{n}$ for some $\mu, \delta>0$. This assumption significantly simplifies the calculation and allows us to state the main conclusions in a clean and transparent fashion. All of our results generalize to nonhomogeneous case, with the cost of more convoluted algebra and more obscure interpretation. We study both the 
transmission graph Laplacian matrix and Laplace domain properties of (2). To clear potential confusion, we agree that whenever the adjective Laplacian is used, we refer to quantities related to the Laplacian matrix $L$; and whenever the noun Laplace is used, we refer to notions about the Laplace transform:

$$
\mathcal{L}\{s(t)\}(\tau):=\int_{0}^{\infty} s(t) e^{\tau t} d t
$$

or notions defined in the Laplace domain.

\section{Characterization OF SyStem Response}

In this section, we give a complete characterization of the system response of (2) based on spectral decomposition in the time and Laplace domains.

We first determine the modes of the system (2) - that is, we compute the eigenvalues of the system matrix $A$. Such eigenvalues indicate whether the system is stable and, if it is, how fast the system converges to an equilibrium state.

Theorem 3.1: Let $0=\lambda_{1}<\lambda_{2} \leq \cdots \leq \lambda_{n}$ be the eigenvalues of $L$ with corresponding orthonormal eigenvectors $v_{1}, v_{2}, \ldots, v_{n}$. Then:

1) 0 is an eigenvalue of $A$ of multiplicity $m-n+1$. The corresponding eigenvectors are of the form $[0 ; P]$ with $P \in \operatorname{kernel}(C)$.

2) $-\gamma$ is a simple eigenvalue of $A$ with $\left[M^{-1 / 2} v_{1} ; 0\right]$ as a corresponding eigenvector.

3) For $i=2,3, \ldots, n, \phi_{i, \pm}=\frac{-\gamma \pm \sqrt{\gamma^{2}-4 \lambda_{i}}}{2}$ are eigenvalues of $A$. For any such $\phi_{i, \pm}$, an eigenvector is given by $\left[M^{-1 / 2} v_{i} ; \phi_{i, \pm}^{-1} B C^{T} M^{-1 / 2} v_{i}\right]$.

Proof: Please refer to our online report [19].

When $m-n+1=0$, or equivalently when the network is a tree, item 1) of Theorem 3.1 is understood to mean that the system matrix $A$ does not have 0 as an eigenvalue. We remark that a similar characterization of the system (2) under a different state representation can be found in [20].

Assuming $\gamma^{2}-4 \lambda_{i} \neq 0$ for all $i$, we get $2 n-1$ nonzero eigenvalues of $A$ from item 2) and item 3) of Theorem 3.1, counting multiplicity, which together with the $m-n+1$ multiplicity from item 1) gives $m+n$ eigenvalues as well as $m+n$ linearly independent eigenvectors. Therefore, we know $A$ is always diagonalizable over the complex field $\mathbb{C}$, provided critical damping-that is $\gamma^{2}-4 \lambda_{i}=0$ for some $i$, does not occur. We assume this is the case in all following derivations. When critical damping does occur, our results can be generalized using the standard Jordan decomposition.

Theorem 3.1 explicitly reveals the impact of the transmission network connectivity as captured by its Laplacian eigenvalues on the system (2) and tells us that the system mode shape is closely related to the corresponding Laplacian eigenvectors. In particular, we note that the real parts of $\phi_{i, \pm}$ are nonnegative, from which we deduce the following corollary.

Corollary 3.2: The system (2) is marginally stable, with marginal stable states of the form $[0 ; P]$ with $P \in \operatorname{kernel}(C)$. Therefore, the system (2) is asymptotically stable on a tree.
The kernel of $C$ corresponds to the set of branch flow vectors $P$ such that $\sum_{j \in N(i)} P_{i j}=0$ for all $i \in \mathcal{N}$. They can be interpreted as flows that are balanced at all the buses (e.g., circulation flows on a loop) for which each bus $i$ is neither a source node (for which $\sum_{j \in N(i)} P_{i j}>0$ ) nor a sink node (for which $\left.\sum_{j \in N(i)} P_{i j}<0\right)$. This corollary tells us that the only possible signals that can persist in (2) are the balancing branch flows. Of course, such marginally stable flows cannot exist in a real system because of losses in transmission lines (in which case the network dynamics (1b) are no longer accurate). Even if we take the simplified model (2), as long as the initial branch flow does not belong to $\operatorname{kernel}(C)$, the system (2) under zero input $P^{m}-d=0$ converges to the nominal state.

Next, we determine the system response to a step function. More precisely, we define $s(t):=P^{m}(t)-d(t)$ as the surplus function and compute the frequency trajectory $\omega(t)$ with $s(t)$ as input to (2), assuming $s(t)$ takes constant value $s$ over time. Note that the components $s_{j}$ can be different over $j$. We put $s=\sum_{i} \hat{s}_{i} M^{1 / 2} v_{i}$ to be the decomposition of $s$ along the scaled Lapalacian eigenvectors (note that the scaling $M^{1 / 2} v_{i}$ is different from the scaling $M^{-1 / 2} v_{i}$ in the following theorem statement).

Theorem 3.3: Let $0=\lambda_{1}<\lambda_{2} \leq \cdots \leq \lambda_{n}$ be the eigenvalues of $L$ with corresponding orthonormal eigenvectors $v_{1}, v_{2}, \ldots, v_{n}$. Assume:

1) The system (2) is initially at the nominal state $x(0)=0$.

2) $\delta^{2}-4 \lambda_{i} \neq 0$ for all $i$.

Then:

$$
\omega(t)=\sum_{i=1}^{n} \frac{\hat{s}_{i}}{\sqrt{\gamma^{2}-4 \lambda_{i}}}\left(e^{\phi_{i,+} t}-e^{\phi_{i,-}}\right) M^{-1 / 2} v_{i}
$$

where:

$$
\phi_{i,+}:=\frac{-\gamma+\sqrt{\gamma^{2}-4 \lambda_{i}}}{2} \quad \phi_{i,-}:=\frac{-\gamma-\sqrt{\gamma^{2}-4 \lambda_{i}}}{2}
$$

Proof: Please refer to our online report [19].

We remark that all conditions in this theorem are for presentation simplicity, and the frequency trajectory (3) can be generalized by adding correction terms to the case where neither condition is imposed. We opt not to do so here because these terms lead to much more complicated notations yet do not reveal any new insights.

This result tells us that the frequency trajectory of (2) can be decomposed along the scaled eigenvectors of the Laplacian matrix $L$. Moreover, we note that all $\phi_{i, \pm}$ have negative real parts except $\phi_{1,+}=0$. Therefore, the only term in (3) that persists is the term involving $\phi_{1,+}$ given as:

$$
\frac{\hat{s}_{1}}{\sqrt{\gamma^{2}-4 \lambda_{1}}} e^{\phi_{1,+}{ }^{t}} M^{-1 / 2} v_{1}=\frac{\hat{s}_{1}}{\gamma} M^{-1 / 2} v_{1}
$$

Thus, under the input $P^{m}-d=s$, the $\omega(t)$ signal converges to the steady state $\frac{\hat{s}_{1}}{\gamma} M^{-1 / 2} v_{1}$ exponentially fast. This allows us to recover the following well-known result in frequencyregulation literature [1], using a new argument. 
Corollary 3.4: In the steady state, the system (2) has synchronized frequencies $\omega_{i}=\omega_{j}=: \omega_{c}$, and $\omega_{c}=0$ if and only if the power injection is balanced $\sum_{i \in \mathcal{N}} s_{i}=0$.

Proof: It is easy to show:

$$
v_{1}=\frac{M^{1 / 2}}{\sqrt{\sum_{j \in \mathcal{N}} M_{j}}} \mathbf{1}_{n}
$$

By Theorem 3.3, we know the steady state of (2) is $\left(\hat{s}_{1} / \gamma\right) M^{-1 / 2} v_{1}$, which then has all entries equal to the same value:

$$
\frac{\hat{s}_{1}}{\gamma \sqrt{\sum_{j} M_{j}}}
$$

Therefore, $\omega_{i}=\omega_{j}=: \omega_{c}$ for all $i, j \in \mathcal{N}$. From $s=$ $\sum_{i} \hat{s}_{i} M^{1 / 2} v_{i}$, we see $\hat{s}_{1}=\left(M^{-1 / 2} s\right)^{T} v_{1}=s^{T} M^{-1 / 2} v_{1}$, and thus:

$$
\begin{aligned}
\sum_{i \in \mathcal{N}} s_{i} & =s^{T} \mathbf{1}_{n}=\sqrt{\sum_{j} M_{j}} s^{T} M^{-1 / 2} v_{1}=\sqrt{\sum_{j} M_{j}} \hat{s}_{1} \\
& =\gamma\left(\sum_{j} M_{j}\right) \omega_{c}=\left(\sum_{j \in \mathcal{N}} D_{j}\right) \omega_{c}
\end{aligned}
$$

Hence, $\omega_{c}=0$ if and only if $\sum_{i \in \mathcal{N}} s_{i}=0$.

It is also informative to look at the system behavior of (2) from the Laplace domain. Instead of analyzing transfer functions from any input to any output as in the classic multi-input-multi-output system analysis, we take a slightly different approach such that the Laplacian matrix spectral information is preserved. More precisely, for a time-variant surplus signal $s(t)$, we first decompose it to the spectral representation $s(t)=\sum_{i=1}^{n} \hat{s}_{i}(t) M^{1 / 2} v_{i}$. Now $\hat{s}_{i}(t)$ is a realvalued signal, and thus assuming it is regular enough, we can write it as the integral of exponential signals $e^{\tau t}$ through inverse Laplace transform. It can be shown that when the input to system (2) takes the form $e^{\tau t} M^{1 / 2} v_{i}$, the steadystate frequency trajectory $\bar{\omega}(t)$ is given by $H_{i}(\tau) e^{\tau t} M^{-1 / 2} v_{i}$, where $H_{i}(\tau)$ is a complex-valued function of $\tau$ specifying the system gain and phase shift. We refer to the function $H_{i}(\tau)$ as the $i$-th spectral transfer function. Compared to classic transfer functions, the spectral version does not capture the relationship between any input-output pair; in contrast, it captures the behavior of system (2) from a network perspective. Once the spectral transfer functions are known, we can compute the steady-state trajectories for the general input signal $s(t)$ through the following synthesis formula:

$$
\bar{\omega}(t)=\sum_{i=1}^{n} \mathcal{L}^{-1}\left\{H_{i}(\tau) \mathcal{L}\left\{\hat{s}_{i}(t)\right\}(\tau)\right\} M^{-1 / 2} v_{i}
$$

Theorem 3.5: For each $i$, assuming $\gamma^{2}-4 \lambda_{i} \neq 0$, the $i$-th spectral transfer function is given by:

$$
H_{i}(\tau)=\frac{\tau}{\tau^{2}+\gamma \tau+\lambda_{i}}
$$

Proof: Please refer to our online report [19].
We remark that a similar formula also shows up in [21] as the representative machine transfer function for swing dynamics.

\section{INTERPRETATIONS}

In this section, we present a collection of intuition we can devise from the results in Section III. They are useful for making general inferences and for the controller design in Section V.

Towards this goal, we rewrite (3) as:

$$
\omega(t)=\sum_{i=1}^{n} \hat{s}_{i} \hat{\omega}_{i}(t) v_{i}
$$

The signal $\hat{\omega}_{i}(t)$ captures the response of system (2) along $v_{i}$ to a step function input. From Section IV-A to IV-D, we focus on $\hat{\omega}_{i}(t)$ and understand how properties of such componentswise responses are related to different system parameters. We then comment on how the discussion can be generalized to the complete trajectory $\omega(t)$ in Section IV-E.

\section{A. Impact of network connectivity}

We first clarify how the network connectivity affects the system. By Theorem 3.1, we see that whether the system oscillates or not is determined by the signs of $\delta^{2}-4 \lambda_{i}$. For $\lambda_{i}$ such that $\delta^{2}-4 \lambda_{i}>0$, we have:

$$
\hat{\omega}_{i}(t)=\frac{1}{\sqrt{\delta^{2}-4 \lambda_{i}}}\left(e^{\phi_{i,+} t}-e^{\phi_{i,-} t}\right) v_{i}
$$

with $\phi_{i, \pm} \leq 0$. Thus the system is overdamped along $v_{i}$, and deviations along $v_{i}$ exponentially fade away without oscillation. The slower-decaying exponential has a decaying rate determined by $\phi_{i,+}$, which is a decreasing function in $\lambda_{i}$. Thus, a larger $\lambda_{i}$ implies faster decaying. Intuitively, this tells us that when the system damping is strong, adding connectivity helps move more disturbances to the damping component so the disturbances can be absorbed sooner.

For $\lambda_{i}$ such that $\delta^{2}-4 \lambda_{i}<0$, we have:

$$
\hat{\omega}_{i}(t)=\frac{1}{\sqrt{\delta^{2}-4 \lambda_{i}}} e^{-\frac{\delta}{2 \mu} t} \sin \left(\frac{\sqrt{4 \lambda_{i}-\delta^{2}}}{2 \mu} t\right) v_{i}
$$

Thus, the system is underdamped along $v_{i}$, and oscillations do occur. We also note that larger values of $\lambda_{i}$ lead to oscillations of higher frequency. This intuitively means that when the system damping is not strong enough, adding connectivity causes the unabsorbed oscillations to propagate throughout the network faster, bringing disturbances to the already overburdened damping components, causing the system to oscillate in a higher frequency.

We thus see that Theorem 3.1 and Theorem 3.3 precisely clarify our seemingly contradicting intuition on whether connectivity is beneficial to stabilization-it depends on how strong the system is damped, i.e., how fast the system can dissipate energy. 


\section{B. Impact of rotational inertia and damping}

Next, we clarify the differences in how the generator inertia and damping impact the system performance. Toward this goal, we examine three common metrics for $\hat{\omega}_{i}(t)$ : a) settling time, which is the time it takes $\hat{\omega}_{i}(t)$ to get within a certain range $^{1}$ around the steady state; $b$ ) nadir, which is defined to be the sup norm of $\hat{\omega}_{i}(t)$; and c) first peak time, which is the time it takes $\hat{\omega}_{i}(t)$ to reach the nadir.

Table I summarizes the formulas ${ }^{2}$ for these metrics. As shown, although the inertia constant, $\mu$, and the damping constant, $\delta$, both capture the "frictional force" of the system, they impact the system performances in different ways. In particular, $\mu$ affects the settling time and first peak time in a linear fashion, but it does not affect the nadir. Intuitively, this means that for a system with larger inertia, the same level of excitation induces a smaller system "acceleration," and thus it takes longer for the system to respond. The invariance of the nadir can be interpreted as a result of energy conservation because the energy dissipation strength is independent of $\mu$.

In contrast, the impact of the damping $\delta$ is less clear from observing the complicated formulas in Table I. Nevertheless, one can show using basic calculus that the settling time and nadir are decreasing functions of $\delta$, and the first peak time is an increasing function of $\delta$. Intuitively, this means that a higher level of damping slows down the system response, but it also increases the energy dissipation rate, leading to a smaller nadir and overall shorter settling time.

\section{Impact of line susceptance}

The line susceptances are implicitly absorbed to the Laplacian eigenvalues $\lambda_{i}$, and its impact is relatively easily shown if we assume that all lines have homogeneous susceptance $\beta$ (that is, $B=\beta I_{m}$ ). Now, let $\bar{\lambda}_{i}$ denote the eigenvalues of $C C^{T}$, which can be interpreted as the pure topological part in the Laplacian spectrum, and we see that $\lambda_{i}=\beta \overline{\lambda_{i}}$. Therefore, the impact of increasing the line susceptance is similar to increasing the overall network connectivity. This agrees with our intuition that a line with smaller susceptance exhibits larger "resistance" to the power flow, and therefore it induces a larger "effective distance" in the topology.

\section{Robustness to disturbance}

The impact of different system parameters in the Laplace domain can be understood from the spectral transfer functions $H_{i}$. In particular, from Theorem 3.5, we can calculate:

$$
\left|H_{i}(\mathrm{j} \sigma)\right|=\frac{|\sigma|}{\sqrt{\mu^{2} \sigma^{4}+\left(\delta^{2}-2 \lambda_{i} \mu\right) \sigma^{2}+\lambda_{i}^{2}}}
$$

For high-frequency signals, the gain can be approximated by $\left|H_{i}(\mathrm{j} \sigma)\right| \approx \frac{1}{\mu \sigma}$, and therefore a system with larger rotational inertia is less sensitive to high-frequency components in its

\footnotetext{
${ }^{1}$ The range is specified as $\left[\omega_{i}^{*}-c, \omega_{i}^{*}+c\right]$, where $\omega^{*}$ is the equilibrium state, and $c$ is a constant.

${ }^{2}$ We define $\Delta_{i}=\left|\delta^{2}-4 \lambda_{i}\right|$ to simplify the formulas. The settling time formula is an upper bound; finding its exact value requires solving transcendental equations and is generally hard.
}

input. The significance of this intuition is twofold. First, it implies that a system with a large $\mu$ value is generally more robust against measurement noise because noise is usually of high frequency. Second, it implies that the only effective way to suppress fluctuations with high frequency (for example, from renewable sources) is to increase the inertia constant $\mu$. Adding damping level $\delta$ or connectivity $\lambda_{i}$, although helpful, would be much less effective.

Equation (4) shows that for any fixed frequency $\sigma,\left|H_{i}(\mathrm{j} \sigma)\right|$ is decreasing in $\delta$. This means that a larger damping leads to smaller gains for all frequencies. Such decrease is negligible for large $\sigma$, and therefore increasing $\delta$ mostly helps the system to suppress low-frequency oscillations. This agrees with our time domain result in (3), which says that the steady-state value of the frequency deviation is inversely proportional to the damping level $\delta$ (and independent of the inertia $\mu$ ). Through a similar argument, we can also see that increasing the network connectivity $\lambda_{i}$ also mostly helps the system suppress lowfrequency oscillations.

\section{E. General inferences}

The complete trajectory (3) is a linear combination of $\hat{\omega}_{i}(t)$, and because of the possibility of negative $\hat{s}_{i}$, our previous discussions do not generalize to $\omega(t)$ in a straightforward way. Instead of focusing on $\omega(t)$ for a specific $s(t)$, we can look at all possible $\omega(t)$ and generalize our previous interpretations to the worst-case performance metric. To be concrete, let us take nadir as an example. By (3), we see the nadir of $\omega(t)$ satisfies:

$$
\begin{aligned}
\|\omega(t)\|_{\infty} & \leq \sum_{i=1}^{n}\left|\hat{s}_{i}\right|\left\|\hat{\omega}_{i}(t)\right\|_{\infty} \leq \sqrt{\sum_{i=1}^{n}\left|\hat{s}_{i}\right|^{2}} \sqrt{\sum_{i=1}^{n}\left\|\hat{\omega}_{i}(t)\right\|_{\infty}^{2}} \\
& =\left(\sqrt{\sum_{i=1}^{n}\left\|\hat{\omega}_{i}(t)\right\|_{\infty}^{2}}\right)\|s\|_{2}:=\|\omega\|_{\infty}^{w}\|s\|_{2}
\end{aligned}
$$

It is easy to see that all the inequalities above can attain equalities. Therefore, among all input $s$ with unit energy $\|s\|_{2}=1$, the worst possible nadir is $\|\omega\|_{\infty}^{w}$, which is a decreasing function of $\delta$, as shown from our previous discussions.

This worst-case nadir is a system level metric that is independent of the input. Although this metric does not predict the exact nadir for any specific input, it does reveal to what extent the system can tolerate disturbances of certain energy, which is a property that is intrinsic to the system itself. Moreover, for the secure and robust operation of the grid, we need to make sure that the worst-case nadir is well-controlled.

Similarly, denoting the settling time of $\hat{\omega}_{i}(t)$ by $t_{i}$, the worst-case settling time of $\omega(t)$ is $\left(\max _{i} t_{i}\right) /\|s\|_{2}$. We can then generalize our previous discussion to conclude that this worst-case metric is a decreasing function of $\delta$ and increasing function of $\mu$. The situation for the first peak time is trickier, and our prediction for $\omega(t)$ based on componentwise $\hat{\omega}_{i}(t)$ usually does not agree with simulations (for example, from [10], [11]). As a result, we expect our previous discussion on 
TABLE I

System Performance in Terms of Network Mixing Capability, Generator inertia, And Damping.

\begin{tabular}{|c|c|c|c|}
\hline Case & Settling Time & Nadir & First Peak Time \\
\hline$\delta^{2}>4 \lambda_{i}$ & $\frac{2 \mu}{\delta-\sqrt{\Delta_{i}}} \ln \left(\frac{1}{2 c \sqrt{\Delta_{i}}}\right)$ & $\frac{1}{\sqrt{\Delta_{i}}}\left[\left(\frac{\delta+\sqrt{\Delta_{i}}}{\delta-\sqrt{\Delta_{i}}}\right)^{\frac{-\delta+\sqrt{\Delta_{i}}}{2 \sqrt{\Delta_{i}}}}-\left(\frac{\delta+\sqrt{\Delta_{i}}}{\delta-\sqrt{\Delta_{i}}}\right)^{\frac{-\delta-\sqrt{\Delta_{i}}}{2 \sqrt{\Delta_{i}}}}\right]$ & $\frac{\mu}{\sqrt{\Delta_{i}}} \ln \left(\frac{\delta+\sqrt{\Delta_{i}}}{\delta-\sqrt{\Delta_{i}}}\right)$ \\
\hline$\delta^{2}<4 \lambda_{i}$ & $\frac{2 \mu}{\delta} \ln \left(\frac{1}{c \sqrt{\Delta_{i}}}\right)$ & $\frac{1}{\sqrt{\Delta_{i}}} \exp \left(-\frac{4 \pi \delta}{\sqrt{\Delta_{i}}}\right)$ & $\frac{4 \pi \mu}{\sqrt{\Delta_{i}}}$ \\
\hline
\end{tabular}

the first peak time to be correct in practice only if the system input is mostly along the direction of a particular $v_{i}$.

\section{F. System trade-offs}

From our previous discussions, we see that when choosing system parameters, there are usually trade-offs among different design goals, and we must balance different aspects to obtain a good design.

Example 4.1 (Trade-off between robustness against measurement noise and system responsiveness): We have shown that in order for the system (2) to be robust against measurement noise, we need an inertia $\mu$ that is large enough. On the other hand, increasing $\mu$ leads to a larger settling time, thus making the system less responsive.

Example 4.2 (Trade-off between small intrinsic frequency and robustness against attack): It is easy to show that $\left|H_{i}(\mathrm{j} \sigma)\right|$ is maximized at $\sigma_{i}^{*}=\sqrt{\frac{\lambda_{i}}{\mu}}$. In other words, the system (2) can be interpreted as a filter around $\sigma_{i}^{*}$ along $v_{i}$, and harmonics around $\sigma^{*}$ are amplified by the network. To decrease the system gain to oscillations of high frequency, we want a smaller $\sigma_{i}^{*}$ and thus smaller $\lambda_{i}$; however, a smaller $\lambda_{i}$ suggests lower connectivity. In fact, one can show that among all possible topologies over $n$ buses, the line topology minimizes $\lambda_{n}$, the largest Laplacian eigenvalue. Such networks are very prone to component failures and fragile to most types of attack.

\section{Controller Design for LoAd-Side Participation}

In this section, we quantify the benefits of integrating loadside participation and demonstrate how we can improve the controller design in a systematic way.

\section{A. Benefits of load-side participation}

We adopt the controller design from [10] as an example to explain the benefits of load-side participation. We assume the system deviation is small so that the capacity bounds of the load-side controllers are not binding. In this setting, the control law of [10] is simplified to:

$$
d_{i}=K_{p} \omega_{i}
$$

which when plugged into (2) can be absorbed into the damping term $\delta \omega_{i}$. Therefore, the integration of controller (5) effectively increases the system damping level. Based on our discussion in Section IV, we then conclude that load-side participation decreases both the settling time and nadir of (2). This means that with load-side participation, system (2) is more responsive and its transient behavior is improved.
Such benefits have been observed and confirmed during a series of work [9]-[12], [22] in their simulations. With our framework, we can theoretically derive such benefits and quantify how beneficial the load-side integration can be when we use a certain system gain, $K_{p}$. Moreover, it is observed in [22] that load-side participation also helps maintain the system stability when the generator output fluctuates. Using our characterization in the Laplace domain, we see that such benefit comes from the improved system ability in suppressing oscillations of relatively low frequency.

\section{B. Proportional-derivative controller}

Despite the many benefits of load-side controllers explained so far, one key component still missing is that they only affect the system damping but cannot increase the system inertia. As mentioned in Section IV, the system inertia is the key parameter affecting the system robustness against highfrequency oscillations. We cannot expect a low-inertia grid with extensive penetration levels of renewable generation to work well without finding a way to produce inertia because renewables introduce more fluctuations to the grid. Nevertheless, a quick glimpse into (2) suggests that to have larger $\mu$, it suffices to add a derivative term in (5):

$$
d_{i}=K_{p} \omega_{i}+K_{d} \dot{\omega}_{i}
$$

This, of course, comes with the price of increased settling time compared to (5), which can be resolved by using a larger $K_{p}$.

Although it is a natural idea to generalize proportional controllers to proportional-derivative (PD) controllers, we see that the necessity of adding the derivative term can actually be reversed engineered from our characterizations. Moreover, our framework reveals how the parameters $K_{p}$ and $K_{d}$ affect the system performance precisely, allowing us to optimize such gains subject to different design goals. Using derivative terms in controller design is often problematic in practice because of the amplified noise in their measurements; however, based on our previous discussions, to improve the grid stability under high-frequency fluctuations, we must have some components of the network that are able to measure the signal derivative either explicitly or implicitly and provide the necessary inertia. From a pure control system point of view, the difficulty of handling high-frequency fluctuations from renewables lies precisely in the accurate measurement of frequency derivatives.

\section{EVALUATion}

In this section, we simulate the controller design (6) using the IEEE 39-bus New England interconnection system, as 


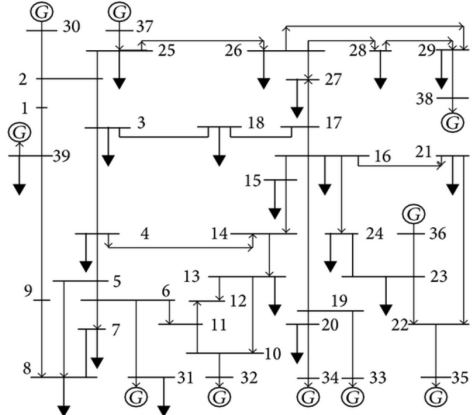

Fig. 1. Line diagram of the IEEE 39-bus interconnection test bed.

shown in Figure 1, and compare its performance to that of (5) and the conventional droop control. There are 10 generators and 29 load nodes in the system, and we take the system parameters from the Matpower Simulation Package [23]. In contrast to our theoretical analysis, the simulation data have heterogeneous inertia and damping. The droop control is implemented as the $D_{j} \omega_{j}$ term for the generator buses and is deactivated for simulations with the controllers (5) and (6). We assume that all the buses (including the generator buses) have load-side participation enabled and pick the controller gains $K_{p}$ and $K_{d}$ heterogeneously in proportional to the bus damping $D_{j}$.

\section{A. Robustness against measurement noise}

We first look at the controller performances against measurement noise. Toward this goal, we add a white Gaussian measurement noise of power $-20 \mathrm{dBW}$ to the frequency sensor at Bus 30, and we observe its frequency trajectory, which is shown in Figure 2. As shown, the controller (5) is less prone to measurement noise compared to the conventional droop control, because it increases the system damping level and therefore helps suppress the low-frequency part of the noise; however, its benefit in suppressing high-frequency noise is limited, shown from its performance gap compared to the controller (6). To more clearly see such distinction, we replace the measurement noise at Bus 30 with the signal $0.2 \sin (10 \pi t)$ pu that contains only the high-frequency component, and we observe its trajectory, which is shown in Figure 3. In this case, we see that controller (5) performs nearly the same as the conventional droop control, whereas the system under the improved controller (6) exhibits a much smaller oscillation.

\section{B. Wind power data}

Next, we look at the performance of the controllers under real wind power generation data from [24]. We choose Bus 30 to be the wind generator, whose output follows the profile given in [24], and we look at the frequency trajectory at Bus 36. The two buses are specifically chosen to be geographically far away so that the simulation results reflect the end user perception of such penetration levels of renewable generation. The simulation results are shown in Figure 4. Compared to controller (5), the improved controller (6) incurs a smaller

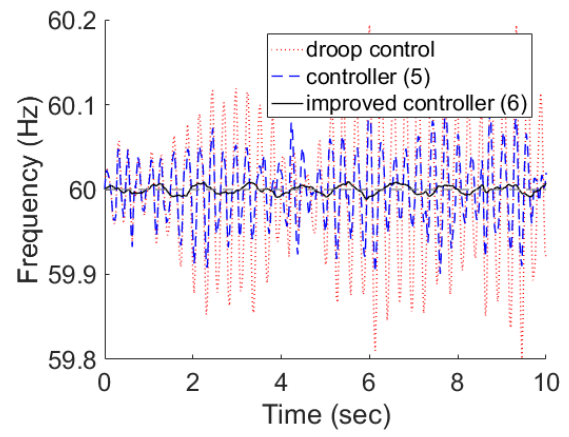

Fig. 2. Frequency trajectory at Bus 30 when we add white Gaussian measurement noise of $-20 \mathrm{dBW}$.

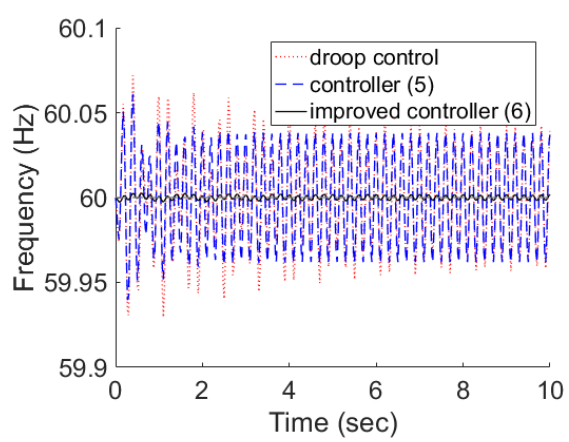

Fig. 3. Frequency trajectory at Bus 30 when we add a signal following the sine curve $0.2 \sin (10 \pi t)$ pu.

frequency deviation almost all time, and the resulting trajectory is smoother. This is because (6) filters high-frequency fluctuations in the generator profile. We expect such benefits to be more significant when the system aggregate load fluctuates more frequently because of increasing penetration levels of renewable generation.

\section{CONCLUSION}

In this work, we proposed a framework using spectral graph theory that captures the interplay among different system parameters. It leads to precise characterizations on how control parameters affect the system performance, and it allows us

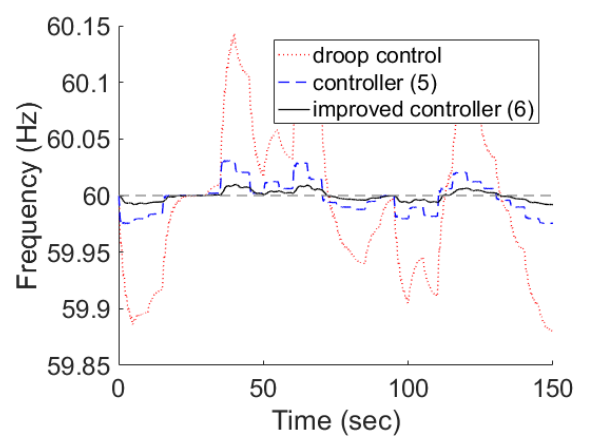

Fig. 4. Frequency trajectory at Bus 36 under wind power output at bus 30 . 
to make general inferences without extensive simulations. We quantified the benefits of load-side participation within this framework and explained how we can improve it so that the system is more robust against high-frequency oscillations.

We remark that our framework can be generalized to include secondary frequency controllers. In particular, load-side controllers for secondary frequency regulation can usually be locally interpreted as the control law (5) plus a term that captures the overall supply-demand imbalance from other parts of the network. Therefore, in terms of system stabilization, our discussion about how load-side participation helps system (2) in both the transient and steady states will still apply. In terms of driving the system back to the nominal state, the framework explains how the cyber and physical network topologies interact with each other and suggests methods to improve the overall system convergence rate. Because of space limitations, we refer interested readers to [25] for more detailed discussions. We are still investigating how our results can be generalized to more detailed models (e.g., where the generators have higher order or nonlinear dynamics).

\section{REFERENCES}

[1] J. Machowski, J. W. Bialek, and J. R. Bumby, Power system dynamics: stability and control. Chichester, U.K.: Wiley, 2008.

[2] I. Hiskens and M. Pai, "Hybrid systems view of power system modelling," in Circuits and Systems, 2000. Proceedings. ISCAS 2000 Geneva. The 2000 IEEE International Symposium on, vol. 2. IEEE, 2000, pp. 228-231.

[3] B. J. Kirby, "Spinning reserve from responsive loads," Report of Oak Ridge National Laboratory, 2003.

[4] PNNL, "Grid friendly controller helps balance energy supply and demand." [Online]. Available: http://readthis.pnl.gov/MarketSource/ ReadThis/B3099_not_print_quality.pdf

[5] D. Trudnowski, M. Donnelly, and E. Lightner, "Power-system frequency and stability control using decentralized intelligent loads," in 2005/2006 IEEE/PES Transmission and Distribution Conference and Exhibition, May 2006, pp. 1453-1459.

[6] J. A. Short, D. G. Infield, and L. L. Freris, "Stabilization of grid frequency through dynamic demand control," IEEE Transactions on power systems, vol. 22, no. 3, pp. 1284-1293, 2007.

[7] D. S. Callaway and I. A. Hiskens, "Achieving controllability of electric loads," Proceedings of the IEEE, vol. 99, no. 1, pp. 184-199, 2011.

[8] A. Molina-Garcia, F. Bouffard, and D. S. Kirschen, "Decentralized demand-side contribution to primary frequency control," IEEE Transactions on Power Systems, vol. 26, no. 1, pp. 411-419, 2011.

[9] C. Zhao, U. Topcu, and S. Low, "Swing dynamics as primal-dual algorithm for optimal load control," in Smart Grid Communications (SmartGridComm), 2012 IEEE Third International Conference on. IEEE, 2012, pp. 570-575.

[10] C. Zhao, U. Topcu, N. Li, and S. Low, "Design and stability of load-side primary frequency control in power systems," Automatic Control, IEEE Transactions on, vol. 59, no. 5, pp. 1177-1189, 2014.

[11] C. Zhao, E. Mallada, S. Low, and J. Bialek, "A unified framework for frequency control and congestion management," in Power Systems Computation Conference (PSCC), 2016. IEEE, 2016, pp. 1-7.

[12] E. Mallada, C. Zhao, and S. Low, "Optimal load-side control for frequency regulation in smart grids," in Communication, Control, and Computing (Allerton), 2014 52nd Annual Allerton Conference on. IEEE, 2014, pp. 731-738.

[13] A. Kasis, E. Devane, C. Spanias, and I. Lestas, "Primary frequency regulation with load-side participation part i: stability and optimality," IEEE Transactions on Power Systems, vol. PP, no. 99, pp. 1-1, 2016.

[14] A. Kasis, E. Devane, and I. Lestas, "Stability and optimality of distributed schemes for secondary frequency regulation in power networks," in 2016 IEEE 55th Conference on Decision and Control (CDC), Dec 2016, pp. 3294-3299.
[15] E. Mallada, "idroop: A dynamic droop controller to decouple power grid's steady-state and dynamic performance," in Decision and Control (CDC), 2016 IEEE 55th Conference on. IEEE, 2016, pp. 4957-4964.

[16] R. Pates and E. Mallada, "Decentralized robust inverter-based control in power systems," arXiv preprint arXiv:1612.05812, 2016.

[17] D. K. Molzahn, F. Drfler, H. Sandberg, S. H. Low, S. Chakrabarti, R. Baldick, and J. Lavaei, "A survey of distributed optimization and control algorithms for electric power systems," IEEE Transactions on Smart Grid, vol. PP, no. 99, pp. 1-1, 2017.

[18] F. R. K. Chung, Spectral Graph Theory. American Mathematical Society, 1997.

[19] L. Guo, C. Zhao, and S. H. Low, "Graph laplacian spectrum and primary frequency regulation," arXiv preprint, 2017.

[20] T. Coletta and P. Jacquod, "Performance measures in electric power networks under line contingencies," arXiv preprint arXiv:1711.10348, 2017.

[21] F. Paganini and E. Mallada, "Global performance metrics for synchronization of heterogeneously rated power systems: The role of machine models and inertia," in 2017 55th Annual Allerton Conference on Communication, Control, and Computing (Allerton), Oct 2017, pp. 324 331.

[22] C. Zhao, U. Topcu, and S. H. Low, "Frequency-based load control in power systems," in American Control Conference (ACC), 2012. IEEE, 2012, pp. 4423-4430.

[23] R. D. Zimmerman, C. E. Murillo-Sánchez, and R. J. Thomas, "Matpower: Steady-state operations, planning, and analysis tools for power systems research and education," IEEE Transactions on power systems, vol. 26, no. 1, pp. 12-19, 2011.

[24] Z. Xu, J. Ostergaard, and M. Togeby, "Demand as frequency controlled reserve," IEEE Transactions on Power Systems, vol. 26, no. 3, pp. 1062 1071, Aug 2011.

[25] L. Guo, C. Zhao, and S. Low, "Cyber network design for secondary frequency regulation: A spectral approach," in Power Systems Computation Conference (PSCC), 2018. IEEE, 2018. 